\title{
Superselective arterial embolization with drug-loaded microspheres for the treatment of unresectable breast cancer
}

\author{
Zhiheng Wang", Huimin Niu", Zhiyong Li, Jie Zhang, Longiin Sha, Qian Zeng, Xia Liu, Jintang Huang \\ Hainan Cancer Hospital, Haikou 570000, China \\ Contributions: (I) Conception and design: Z Wang, H Niu; (II) Administrative support: None; (III) Provision of study materials or patients: None; \\ (IV) Collection and assembly of data: Z Li, L Sha, Q Zeng, J Zhang, X Liu, J Huang; (V) Data analysis and interpretation: None; (VI) Manuscript \\ writing: All authors; (VII) Final approval of manuscript: All authors. \\ \#These authors contributed equally to this work as co-first authors. \\ Correspondence to: Huimin Niu. Hainan Cancer Hospital, Haikou 570000, China. Email: 604646525@qq.com.
}

\begin{abstract}
Background: To investigate the short-term efficacy and safety of drug-eluting bead transcatheter arterial chemoembolization (DEB-TACE) using CalliSpheres ${ }^{\circledR}$ microspheres in the treatment of unresectable locally advanced breast cancer (LABC).

Methods: DEB-TACE using CSM was performed in 15 patients with LABC after failure of medical treatment. The efficacy was evaluated based on the modified Response Evaluation Criteria in Solid Tumors (mRECIST). The postoperative adverse reactions and complications were analyzed. The changes of white blood cell (WBC) count, creatine kinase isoenzyme-MB (CK-MB), B-type natriuretic peptide (BNP), and carbohydrate antigen15-3 (CA15-3) before and after treatment were compared by using Wilcoxon signed-rank test.

Results: The surgeries were successful in all patients. The subjects were followed up for 2-60 months (median: 10 months). According to the mRECIST, no patient achieved complete remission (CR) 1, 3, and 5 months after surgery, and partial response (PR) was achieved in 9, 11, and 11 cases; also, there were 6, 4, and 2 stable disease (SD) cases, and 0,0, and 2 progressive disease (PD) cases. The postoperative WBC count, CK-MB level, and BNP level were not significantly different from those before surgery, whereas the CA15-3 level significantly decreased. The main postoperative adverse reactions were pain, fever, and gastrointestinal reactions. No severe adverse reactions were observed.
\end{abstract}

Conclusions: DEB-TACE with CalliSpheres ${ }^{\circledR}$ microspheres is a safe and feasible treatment for LABC. However, more multi-center studies with larger sample sizes are still warranted.

Keywords: Locally advanced breast cancer (LABC); CalliSpheres ${ }^{\circledR}$ drug-loaded microspheres; safety; effectiveness; chemoembolization

Submitted Oct 24, 2019. Accepted for publication Nov 04, 2019.

doi: 10.21037 /gs.2019.12.06

View this article at: http://dx.doi.org/10.21037/gs.2019.12.06

\section{Introduction}

Breast cancer is the most common malignant tumor in Chinese women, with an incidence rate of 28.77 per 100,000 persons and a mortality rate of 6.35 per 100,000 persons (ranking first and second place, respectively, among malignancies in women). Locally advanced breast cancer (LABC) refers to bulky invasive breast tumors without distant metastasis that are difficult to resect. More than $90 \%$ of LABC cases are associated with regional lymph node metastasis (1). LABC accounts for about $20-30 \%$ of new breast cancer cases in China. It is mainly treated with taxanes and anthracyclines, and the overall prognosis is poor (2). Transarterial chemoembolization with drug-eluting beads (DEB-TACEs) kills tumor cells via the slow release of chemotherapy drugs after embolization of the tumor area. Its efficacy and safety in treating liver cancer has been well demonstrated $(3,4)$. CalliSpheres ${ }^{\circledR}$ microsphere is a domestic drug-loading embolization material. While its role in treating 


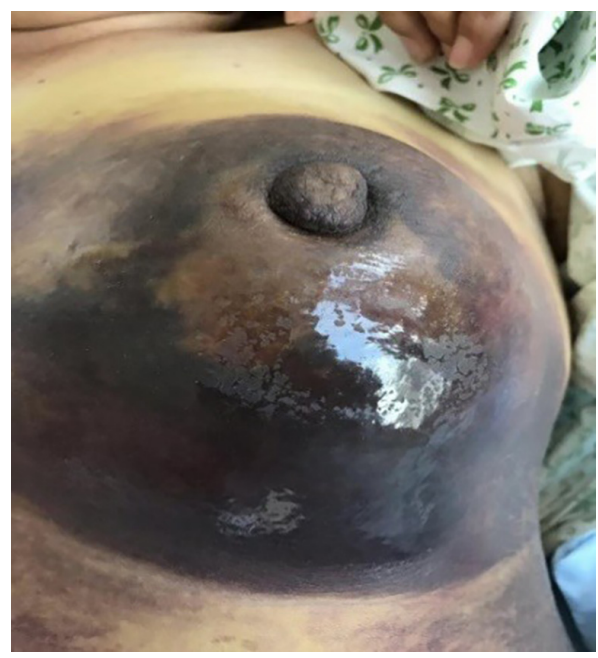

Figure 1 Case 1: before embolization.

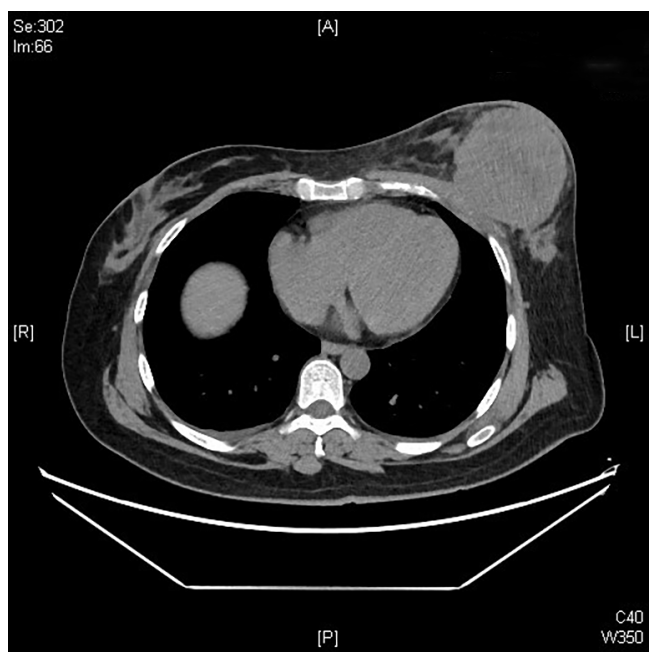

Figure 2 Case 1: CT performed before embolization.

liver cancer has been widely described, its application in the treatment of LABC has rarely been reported. In this article, we summarized our recent experience in treating LABC with CalliSpheres ${ }^{\circledR}$ DEB-TACE.

\section{Methods}

\section{General data}

\section{Patients}

The clinical data of 15 clinically diagnosed LABC patients who had been treated with DEB-TACE in our center from March 2018 to March 2019 were retrospectively analyzed.

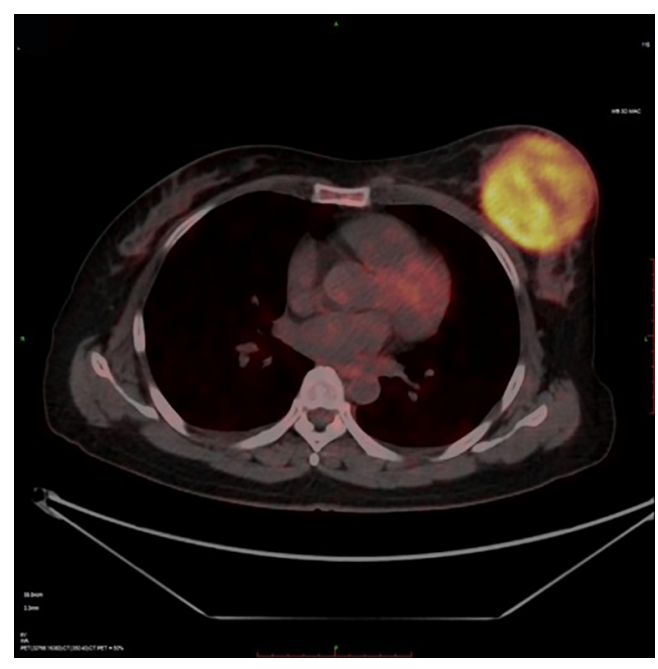

Figure 3 Case 1: PET-CT performed before embolization.

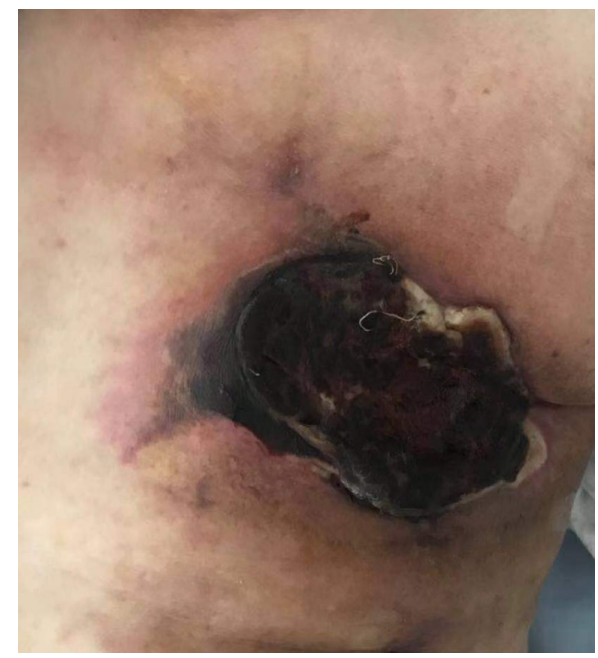

Figure 4 Case 2: before embolization.

All the subjects were female patients aged $42-81$ years (mean: 60.8 \pm 2.3 years). The subjects' tumors, located at either the left side $(\mathrm{n}=9)$ or right side, were sized $10.7 \pm 2.1 \mathrm{~cm}$ $\times 7.5 \pm 1.1 \mathrm{~cm} \times 4.9 \pm 0.8 \mathrm{~cm}$ (Figures $1-4)$. The tumors were pathologically confirmed as invasive ductal carcinomas, accompanied by axillary lymph node metastasis.

\section{Inclusion and exclusion criteria}

The inclusion criteria included the following: (I) pathologically confirmed as LABC; (II) unresectable primary lesion that was unresponsive to medical treatment; (III) with an Eastern Cooperative Oncology Group (ECOG) 


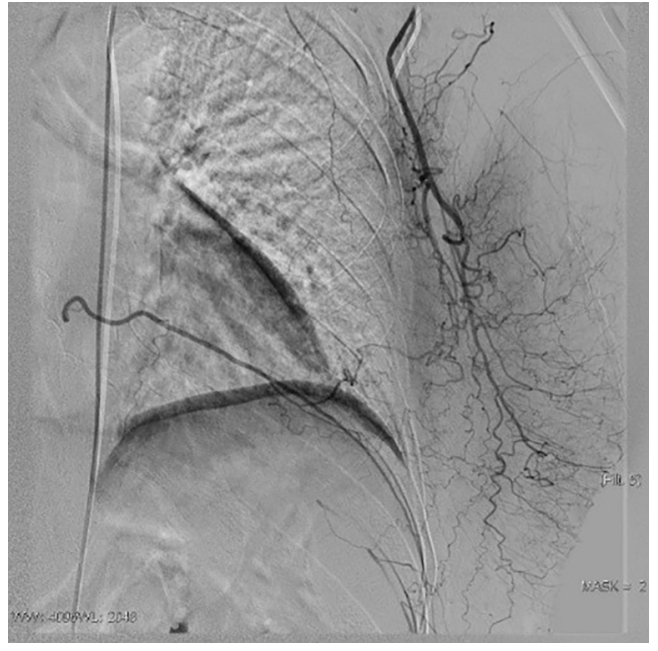

Figure 5 Case 1: feeding artery of breast cancer (a branch of subclavian artery).

score of 0-2; and (IV) with a Child-Pugh class of A or B.

The exclusion criteria included the following: (I) cachexia and multiple organ failure; (II) with an expected survival of $<3$ months; (III) coagulation abnormalities that were difficult to correct; (IV) infection that was difficult to control; and (V) grade 3 or higher bone marrow suppression that was difficult to correct.

\section{Methodology}

Equipment and consumables for interventional surgery The Innova IGS 540 DSA system (GE, USA) was used. The CalliSpheres ${ }^{\circledR}$ beads were produced by Jiangsu Hengrui Medicine Co., Ltd., Jiangsu, China (production license: Jiangsu Food and Drug Administration Production License for Medical Device No. 20090041; product registration certificate number: National Registration Certificate No. 20153771072; and product standard label: TZB/National 4586-2013).

\section{Loading of epirubicin hydrochloride in CalliSpheres ${ }^{\circledR}$ beads}

After 40-80 mg of epirubicin hydrochloride was dissolved in $4 \mathrm{~mL}$ of water for injection or $5 \%$ glucose injection, the CaliliSpheres ${ }^{\circledR}$ drug-loaded beads $(100-300 \mu \mathrm{m})$ were drawn with a $20-\mathrm{mL}$ syringe. The syringe was left standing for 3 minutes, and then the supernatant was discarded. The epirubicin hydrochloride solution was infused into the $20-\mathrm{mL}$ syringe containing the CelliSpheres ${ }^{\circledR}$ beads by a 3 -way

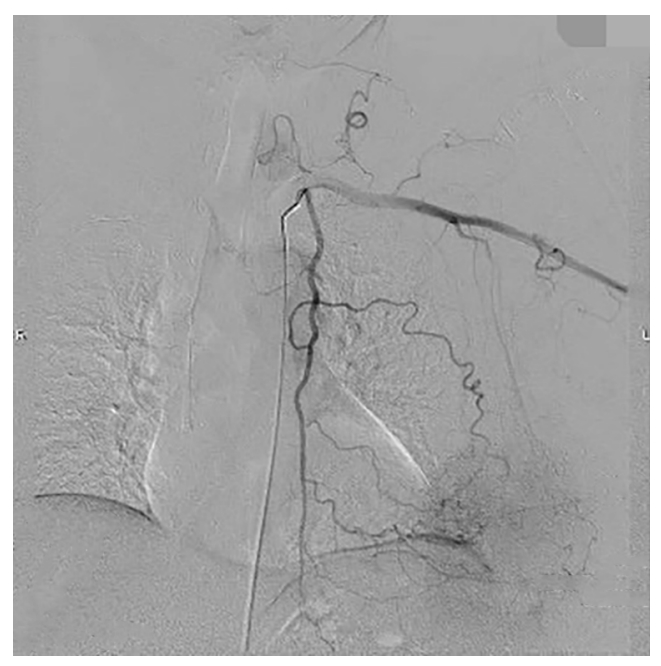

Figure 6 Case 1: feeding artery of breast cancer (a branch of the internal thoracic artery).

stopcock, and these two materials were well mixed by shaking the syringe. The above operation was repeated after the syringe was placed at rest for 5 minutes, and the total resting time was 30 minutes. Finally, a nonionic contrast agent was mixed with the prepared bead liquid in a ratio of 1:1.

\section{Interventional surgical procedure}

An intravenous analgesia pump was installed before surgery and was turned on after the patient arrived at the operating room. The bilateral groin areas were routinely disinfected and draped. The Seldinger technique was applied, during which the femoral artery was punctured with a $5 \mathrm{~F}$ catheter trocar (Radifocus, Terumo, Japan), and a 5 F Bern or Cobra2 catheter (Boston Scientific, USA) was placed in the internal thoracic artery and the iliac artery at the affected side. Digital subtraction angiography (DSA) was performed with a non-ionic contrast agent iodixanol (Suzhou Hengrui Biomedical Technology Co., Ltd.), with a flow rate of $3 \mathrm{~mL} / \mathrm{s}$, a total volume of $12 \mathrm{~mL}$, and a pressure of $300 \mathrm{psi}$. After the feeding artery of the tumor was located, a $2.7 \mathrm{~F}$ torqueable microcatheter (Boston Scientific, USA) was superselected to the tumor-feeding artery and then subtracted, with a flow rate of $3 \mathrm{~mL} / \mathrm{s}$, a total volume of $9 \mathrm{~mL}$, and a pressure of 150 psi. The Callispheres ${ }^{\circledR}$ microspheres loaded with epirubicin hydrochloride were injected to embolize the tumor-feeding artery through the microcatheter. The embolization was terminated when the blood flow in the tumor-feeding artery became stagnant or when vascular casts occurred (Figures 5-12). 


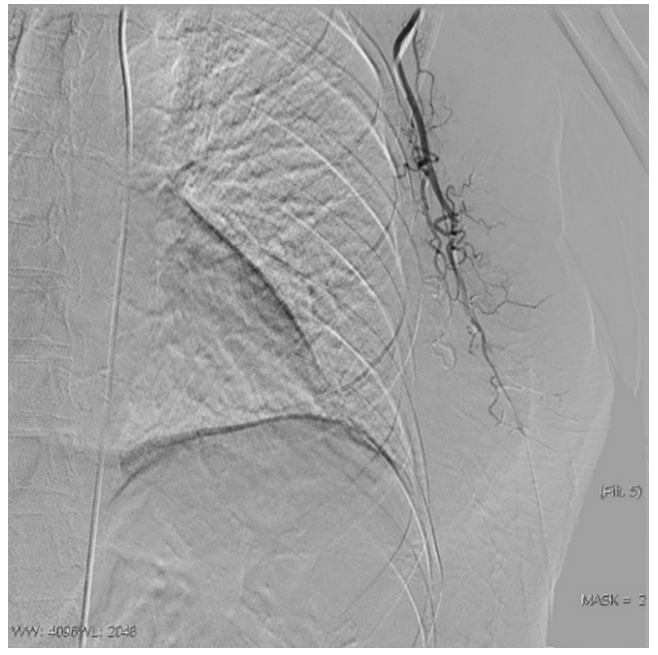

Figure 7 Case 1: after the branch of the subclavian artery was embolized.

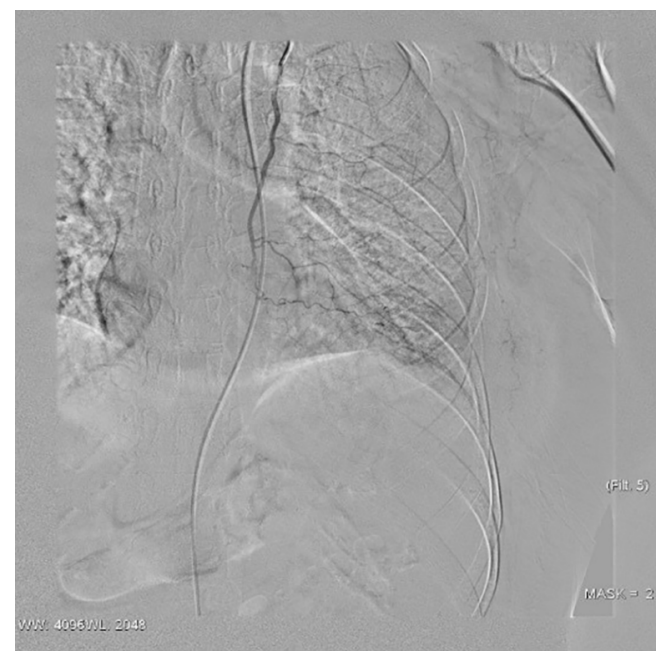

Figure 8 After the branch of internal the thoracic artery was embolized.

\section{Postoperative management}

After the surgery, the changes in white blood cell (WBC) amount, CK-MB, and BNP were monitored. Analgesia, antiemetic, and symptomatic treatment were offered for 3-5 days; if necessary, antibiotics were used to treat secondary infections.

\section{Follow-up}

The changes in CT or PET-CT findings and tumor markers (CA15-3) were compared before and after treatment. The treatment responses were classified as complete

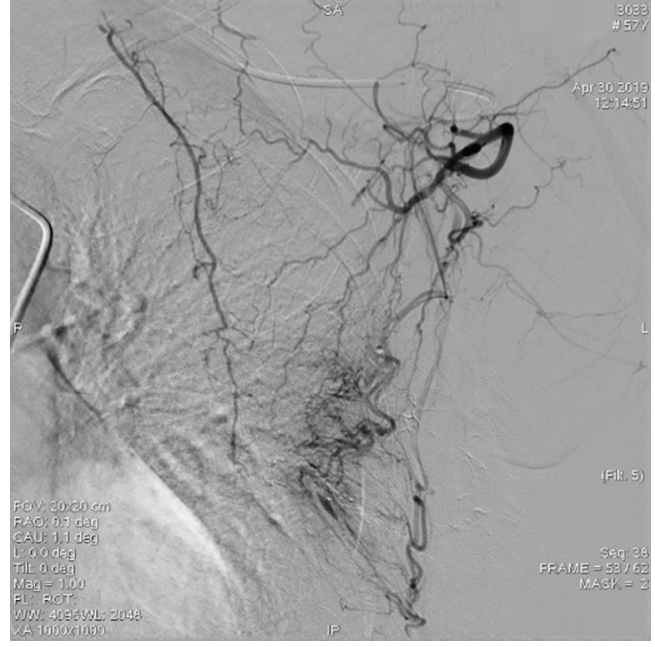

Figure 9 Case 2: feeding artery of breast cancer (a branch of the subclavian artery).

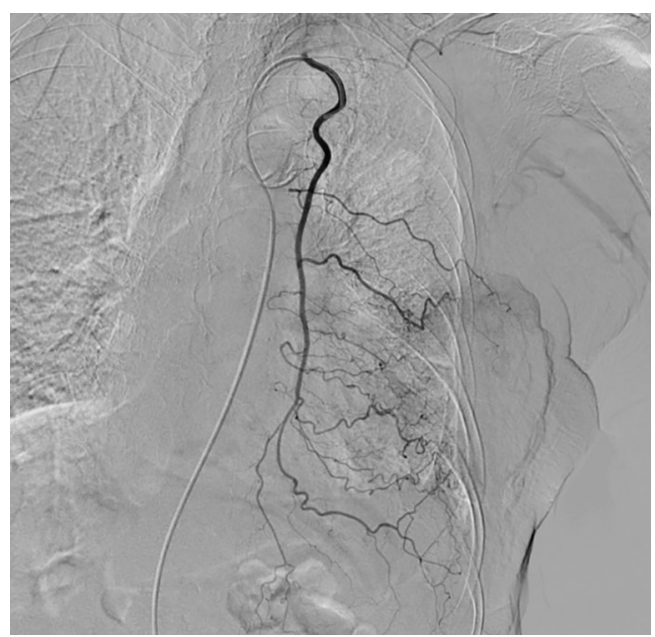

Figure 10 Case 2: feeding artery of breast cancer (a branch of the internal thoracic artery).

remission (CR), partial response (PR), stable disease (SD), or progressive disease (PD) according to the Modified Response Evaluation Criteria in Solid Tumors (mRECIST), and the evaluation was performed 1,3 , and 5 days after surgery. The adverse reactions and complications that occurred during the treatment were recorded and treated.

\section{Statistical analysis}

The statistical analysis was performed using the SPSS 19.0 software package. The changes of WBC count, and CK- 


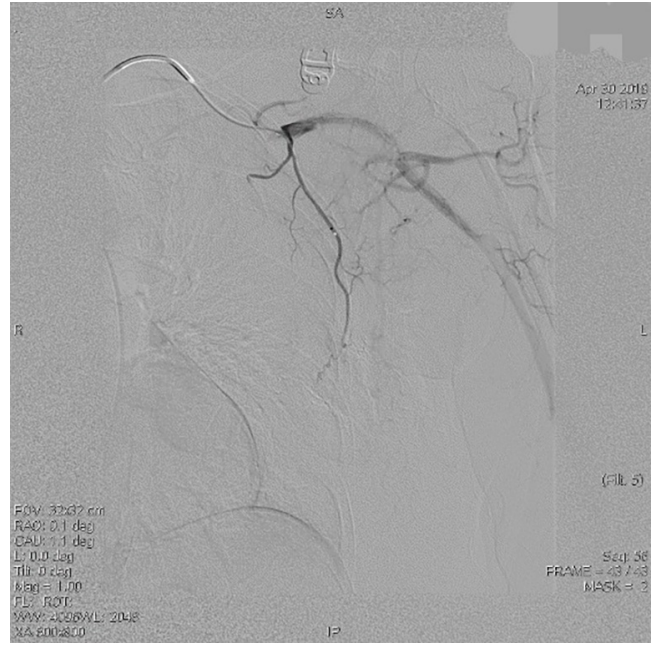

Figure 11 Case 2: after the branch of the subclavian artery was embolized.

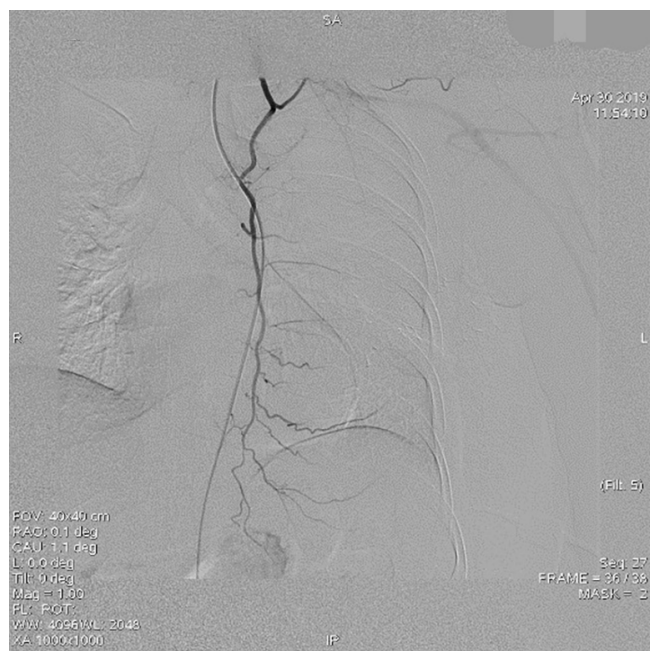

Figure 12 Case 2: after the branch of the internal thoracic artery was embolized.

Table 1 Outcomes of patients during the follow-up period (according to the mRECIST)

\begin{tabular}{lccc}
\hline Outcome & 1 month & 3 months & 5 months \\
\hline CR & 0 & 0 & 0 \\
PR & 9 & 11 & 11 \\
SD & 6 & 4 & 2 \\
PD & 0 & 0 & 2 \\
\hline
\end{tabular}

mRECIST, modified Response Evaluation Criteria in Solid Tumors; CR, complete remission; PR, partial response; SD, stable disease; PD, progressive disease.
$\mathrm{MB}, \mathrm{BNP}$, and CA15-3 levels before and after treatment were compared by using Wilcoxon signed-rank test. A P value of $<0.05$ was considered statistically significant.

\section{Results}

CalliSpheres ${ }^{\circledR}$ DEB-TACE was successfully completed in all 15 patients (100\%). The average hospital stay was 7.8 days. The follow-up lasted 2-16 months, and the median followup duration was 10 months. According to the mRECIST, no patient achieved CR; 9, 11, and 11 patients achieved PR 1,3 , and 5 months after surgery, respectively; the number of SD cases was 6, 4, and 2, and, the number of PD cases was 0,0 , and 2 at the same respective time points (Table 1) (Figures 13-19). All patients survived during the follow-up period. The postoperative WBC count, CK-MB, and BNP were not significantly different from those before surgery, whereas CA15-3 level significantly decreased (Table 2). The main postoperative adverse reactions were pain, fever, and gastrointestinal reactions. All patients suffered from pain in the treatment area: the visual analog scale (VAS) score was 5 in 3 patients and greater than 7 in the remaining 12 patients. All patients experienced fever after surgery, which was moderate in 13 patients and transiently high in 2 patients. The postoperative gastrointestinal reactions were mild in 4 patients and severe in 1 patient. One patient died of heart failure 4 months after surgery.

\section{Discussion}

LABC is mainly treated with chemotherapy, endocrine therapy, and targeted therapy to curb the progression of this deadly disease. However, the average survival time is only $18-30$ months (5) after treatment, and some patients are unable to adhere to the chemotherapy drugs due to the adverse reactions. In an animal study, compared with transarterial administration alone, the drug-loaded microspheres could embolize the tumorfeeding artery and locally offer a sustained and slow release of the chemotherapy drug; the local peak blood drug concentration was reached on day 3 and maintained for 7-14 days. The systemic blood concentration was reduced by $82 \%$ (6), which could effectively reduce the adverse reactions of drugs. Another study showed good safety and a high tumor response rate (7) in liver cancer patients. In recent years, articles on the treatment of liver metastases from breast cancer with DEB-TACE have been published $(8,9)$; however, the application of DEB-TACE for LABC 


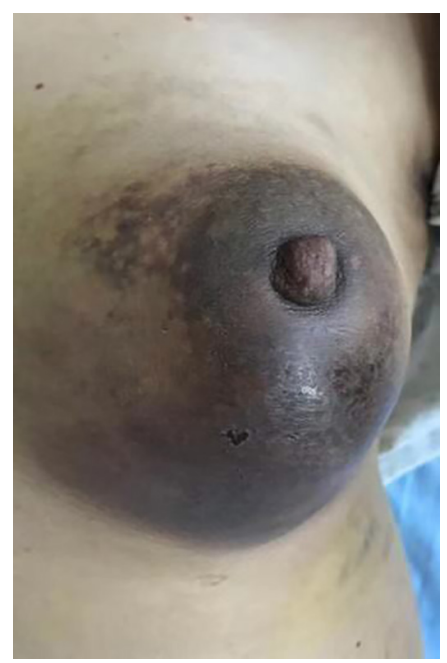

Figure 13 Case1: 1 month after embolization.

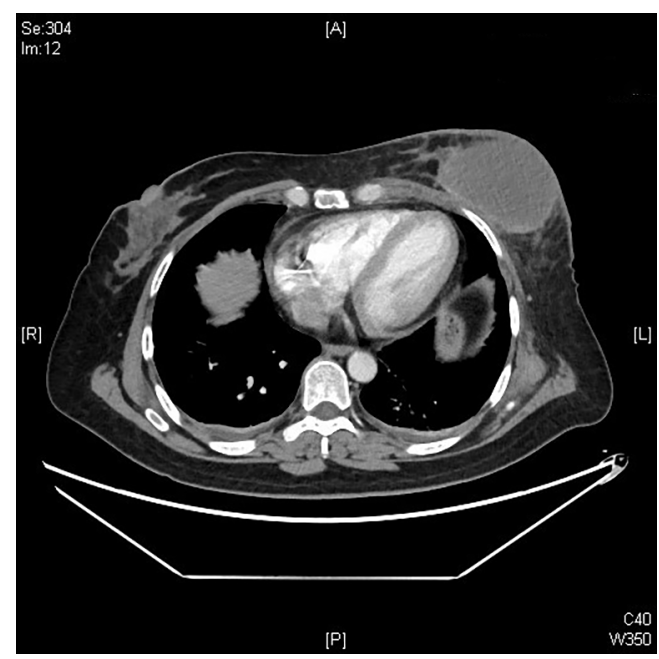

Figure 14 Case 1: CT performed 1 month after embolization.

patients has not been described.

CalliSpheres ${ }^{\circledR}$ drug-loaded microsphere is a new madein-China embolic material. With its main component being the negatively charged polyvinyl alcohol fibers with a diameter of 100-300 $\mu \mathrm{m}$, it has good compressibility and histocompatibility. The attraction between positive and negative charges can load positively charged antitumor drugs such as anthracyclines (epirubicin, pirarubicin, etc.). In our current series, $40-80 \mathrm{mg}$ of epirubicin hydrochloride was loaded into 15 patients according to body surface area and age.

Special attention must be paid during the surgery.

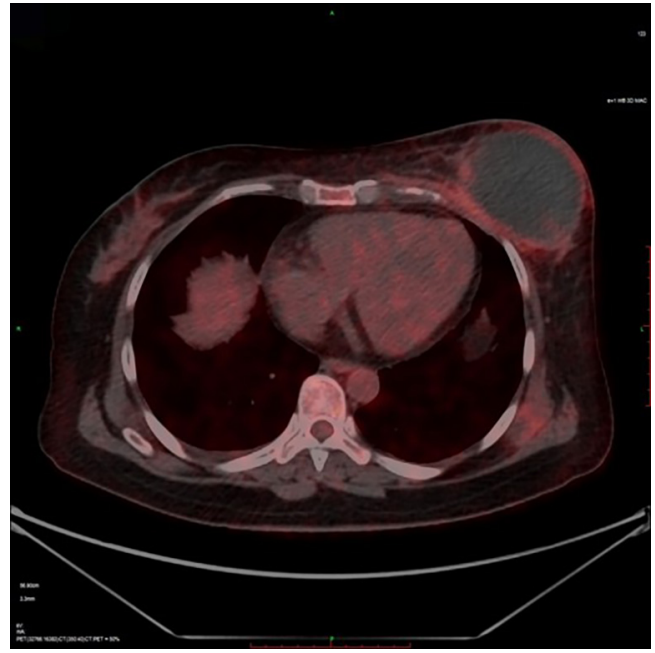

Figure 15 Case 1: PET-CT performed 1 month after embolization.

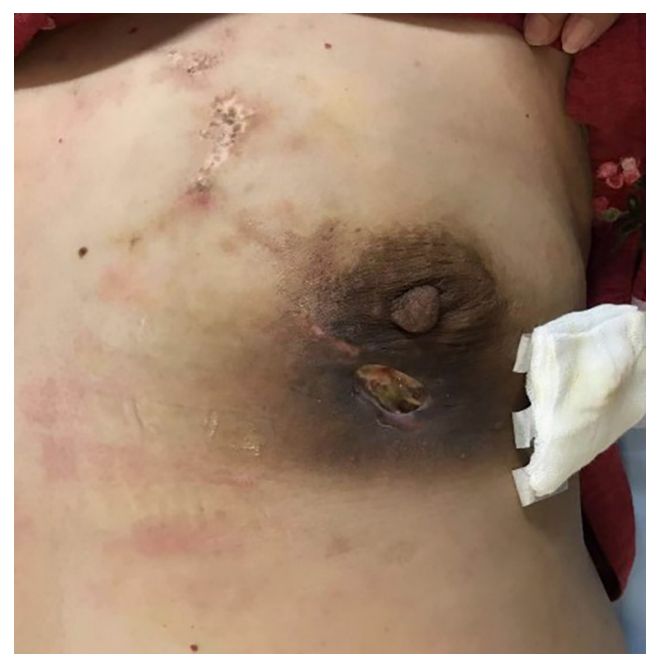

Figure 16 Case 1: 5 months after embolization.

(I) The tumor-feeding artery should be completely embolized whenever possible. The lesions of LABC are often large and diffuse, and over $90 \%$ of such lesions have regional lymph node metastasis (e.g., in the axillary area). Therefore, axillary angiography is often required in addition to the angiography of the internal thoracic artery and intercostal artery. (II) A 5F Bern catheter can be used. After the catheter is placed into the proximal side of the subclavian artery at the affected side, the catheter is inserted upwards (with the head down) and then can directly enter the internal thoracic artery at the affected side. (III) Microcatheters are used for superselective embolization, 


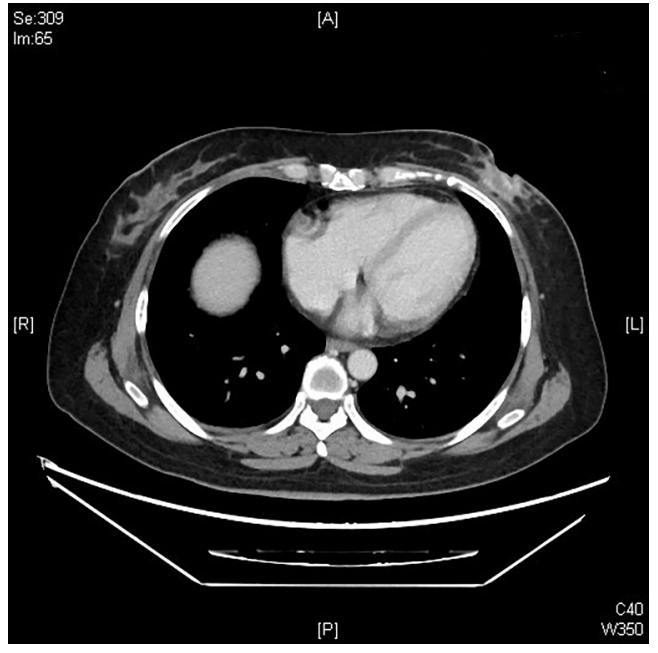

Figure 17 Case 1: CT performed 5 months after embolization.

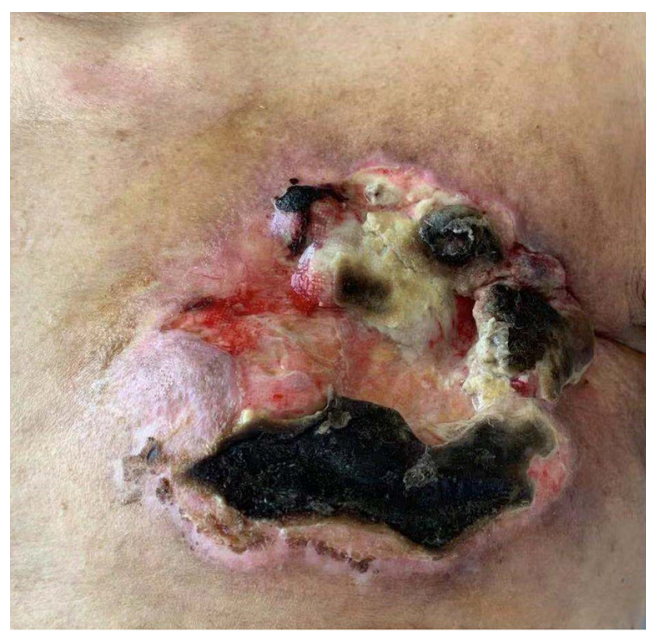

Figure 18 Case 2: 1 month after embolization.

so as to protect normal tissues as much as possible. (IV) For tumors larger than $5 \mathrm{~cm}$, a one-attempt embolization procedure may lead to severe post-embolic syndrome (manifested as severe pain, fever, secondary infection, etc.); therefore, two embolization sessions may be considered to reduce postoperative adverse reactions.

All of our patients suffered from severe pain at the embolization area after surgery, with a VAS score of more than 7 points in most patients. Therefore, the preoperative installation of patient-controlled analgesia (PCA) pump is required. If necessary, an anesthesiologist can be invited to assist the analgesia. Postoperative fever is also common, mainly the absorption fever caused by tumor ischemia

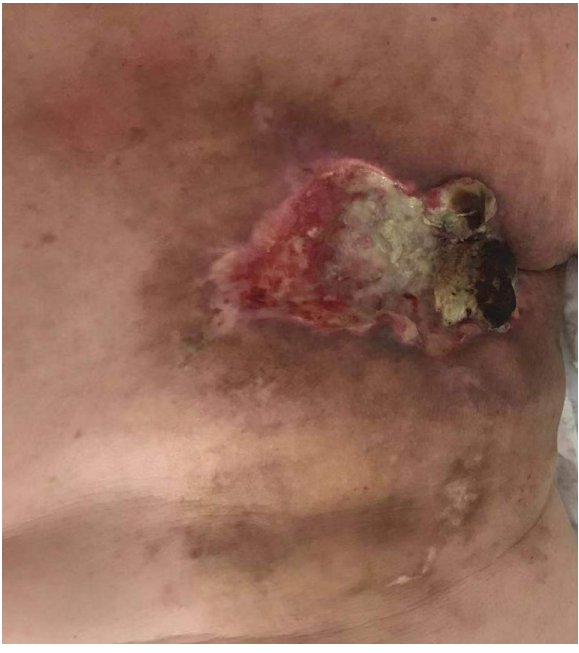

Figure 19 Case 2: 3 months after embolization.

Table 2 Changes in laboratory indicators $(\overline{\mathrm{x}} \pm \mathrm{SD})$

\begin{tabular}{lccc}
\hline Markers & $\begin{array}{c}\text { Before } \\
\text { operation }\end{array}$ & $\begin{array}{c}3 \text { days after } \\
\text { surgery }\end{array}$ & $\begin{array}{c}3 \text { weeks after } \\
\text { surgery }\end{array}$ \\
\hline WBC count $\left(10^{9} / \mathrm{L}\right)$ & $6.6 \pm 1.8$ & $5.8 \pm 1.1$ & $5.0 \pm 1.2$ \\
CK-MB $(\mathrm{U} / \mathrm{L})$ & $10.3 \pm 3.8$ & $13.5 \pm 4.6$ & $14.2 \pm 4.8$ \\
BNP $(\mathrm{pg} / \mathrm{mL})$ & $43.1 \pm 8.6$ & $54.6 \pm 9.9$ & $49.1 \pm 9.4$ \\
CA15-3 $(\mathrm{U} / \mathrm{mL})$ & $77.4 \pm 19.3$ & $69.1 \pm 14.3$ & $35.2 \pm 10.1^{*}$ \\
\hline
\end{tabular}

*, $\mathrm{P}<0.05$. WBC, white blood cell; CK-MB, creatine kinase isoenzyme-MB; BNP, B-type natriuretic peptide; CA15-3, carbohydrate antigen 15-3.

and necrosis. The body temperature should be closely observed if it is within $38.5^{\circ} \mathrm{C}$. If the body temperature rises after 3 days, along with continuous increase of the WBC count, antibiotic treatment may be considered. In some patients, tumor necrosis may cause local skin ulceration and exudation, which requires long-term dressing change. Postoperative gastrointestinal reactions are not uncommon. One of our patients experienced severe nausea and vomiting symptoms, which lasted for more than 1 week. After electrolyte imbalance was alleviated, treatment with metoclopramide and tropisetron hydrochloride failed. Luckily, these symptoms disappeared after aprepitant treatment. Epirubicin has been reported to be cardiotoxic, which may lead to myocardial injury and cardiac insufficiency. Fortunately, no chest pain, chest tightness, or abnormal CK-MB and BNP levels were observed in our series during the perioperative period, which might have 
been due to the use of epirubicin tailored according to the patient's physical condition and age: patients aged 81 years and older were administered with $40 \mathrm{mg}$ of epirubicin hydrochloride, whereas the remaining patients used a full dose $(80 \mathrm{mg})$ of epirubicin hydrochloride. Of course, the absence of cardiotoxicity might also be explained by the small sample size of our current study. One patient in our series suffered from a large amount of pericardial and pleural effusions due to disease progression and died of cardiac failure 4 months after surgery, which was not necessarily related to the use of epirubicin hydrochloride. Several studies have also confirmed that DEB-TACE can reduce the incidences of postoperative adverse reactions and postoperative complications (10-12).

To summarize, our current study has demonstrated that CalliSpheres ${ }^{\circledR}$ DEB-TACE has definite short-term efficacy and is safe and feasible for the clinical treatment of LABC. It offers a new treatment option for patients who cannot be treated surgically. However, more multi-center studies with larger sample sizes are still warranted.

\section{Acknowledgments}

None.

\section{Footnote}

Conflicts of Interest: The authors have no conflicts of interest to declare.

Ethical Statement: The authors are accountable for all aspects of the work in ensuring that questions related to the accuracy or integrity of any part of the work are appropriately investigated and resolved. All patients signed the informed consent forms concerning the use of all case data and figures. The study was approved by the Ethics Committee of our hospital.

\section{References}

1. Lewis RS, George A, Rusby JE. Nipple-sparing mastectomy in women at high risk of developing breast cancer. Gland Surg 2018;7:325-36.

2. Shen ZZ, Liu GY, Su FX, et al. Neoadjuvant chemotherapy with docetaxel plus epirubicin for locally advanced breast cancer: a multi center phase II study. Zhonghua Zhong Liu Za Zhi 2005;27:126-8.
3. Poon RT, Tso WK, Pang RW, et al. A phase I/II trial of chemoembolization for hepatocellular carcinoma using a novel intra-arterial drug-eluting bead. Clin Gastroenterol Hepatol 2007;5:1100-8.

4. Malagari K, Pomoni M, Moschouris H, et al. Chemoembolization of hepatocellular carcinoma with Hepasphere 30-60um. Safety and efficacy study. Cardiovasc Intervent Radiol 2014;37:165-75.

5. Collarino A, Fuoco V, Pereira Arias-Bouda LM, et al. Novel frontiers of dedicated molecular imaging in breast cancer diagnosis. Transl Cancer Res 2018;7:S295-306.

6. Hong K, Khwaja A, Liapi E, et al. New intra-arteral drug delivery system for the treatment of liver cancer: preclinical assessment in a rabbit model of liver cancer. Clin Cancer Res 2006;12:2563-7.

7. Song MJ, Park CH, Kim JD, et al. Drug-eluting bead loaded with doxorubicin versus conventional Lipidolbased transarterial chemoembolization in the treatment of hepatocellular carcinoma: a case-control study of Asian patients. Eur J Gastroenterol Hepatol 2011;23:521-7.

8. Martin RC, Robbins K, Fagés JF, et al. Optimal outcomes for liver-dominant metastatic breast cancer with transarterial chemoembolization with drug-eluting beads loaded with doxorubicin. Breast Cancer Res Treat 2012;132:753-63.

9. Lin YT, Médioni J, Amouyal G, et al. Doxorubicin-Loaded

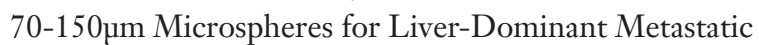
Breast Cancer: Results and Outcomes of a Pilot Study. Cardiovasc Intervent Radiol 2017;40:81-9.

10. Facciorusso A, Mariani L, Sposito C, et al. Drug-eluting beads versus conventional chemoembolization for the treatment of unresectable hepatocellular carcinoma. J Gastroenterol Hepatol 2016;31:645-53.

11. Wu B, Zhou J, Ling G, et al. CalliSpheres drug-eluting beads versus lipidol transarterial chemoembolization in the treatment of hepatocellular carcinoma: a short-term efficacy and safety study. World J Surg Oncol 2018;16:69.

12. Zou JH, Zhang L, Ren ZG, et al. Efficacy and safety of c TACE versus DEB TACE in patients with hepatocellular carcinoma: A meta-analysis. J Dig Dis 2016;17:510-7.

Cite this article as: Wang Z, Niu H, Li Z, Zhang J, Sha L, Zeng Q, Liu X, Huang J. Superselective arterial embolization with drug-loaded microspheres for the treatment of unresectable breast cancer. Gland Surg 2019;8(6):740-747. doi: $10.21037 /$ gs.2019.12.06 